\title{
Taking an Idea from Inception to Innovation: Evolution in Restrictive Transfusion Criteria
}

\author{
Mark K. Wax, MD, FACS, FRCS(C) ${ }^{1}$ \\ ${ }^{1}$ Department of Otolaryngology, Oregon Health \& Science University, \\ Portland, Oregon \\ Facial Plast Surg 2020;36:681-683.
}

\author{
Address for correspondence Mark K. Wax, MD, FACS, FRCS(C), \\ Department of Otolaryngology, Oregon Health \& Science University, \\ 3181 SW SM Jackson Park RD PV-01, Portland, OR 97239 \\ (e-mail: waxm@ohsu.edu).
}

\begin{abstract}
Keywords

- transfusion

- free flap

- hematocrit

- head and neck

Innovation in surgical care is a complex procedure. When you reflect on how your practice has changed, whether it be 5 years or over decades, it can be enlightening to not only see the change but also conceptualize how it came about. Examining one's practice as part of Pittsburgh Sleep Quality Index or as a result of reading the literature, attending a meeting, or some other educational activity can lead one to question if there is a better method available. In this manuscript, I will describe how outside influences initiated a paradigm shift that ultimately benefited patient care, the system, and my practice. The methodology has been used over the course of my career to influence and modulate practice patterns.
\end{abstract}

I would like to thank Dr. Kadakia and Dr. Wimalawansa for inviting me to submit a manuscript on taking an idea from inception to innovation. Perhaps the most complex part of deciding how to approach this subject was reflecting on what significant changes I have introduced into my practice that were not just subjective and anecdotal but were evidencedbased and had the potential to affect patient care; perhaps even instituting a paradigm shift in the management of not only my patients, but also all of our patients. Over the course of a long career, the mark of a successful surgeon is the ability to recognize needed change and to institute that change in one's practice. Oftentimes incremental changes are adapted over a long period. When I reflect on what I did 20 to 30 years ago and what I am doing now, there is a vast difference in many areas. It is the mark of our specialties' continued interest in education and pursuit of excellence that we adapt small incremental changes over time. So when examined in a retrospective nature the change is substantial. It is only after self-reflection and Pittsburgh Sleep Quality Index (PSQI) evaluations that one can look back to see how one's practice parameters have evolved. Dr. Ashok Shaha in the early days of my career cemented that the first 10 years of one's career are spent in learning how to do the procedure, the second 10 years are spent in learning when to do the procedure, and the final 10 years are spent learning when not to do the procedure.

I have chosen to address the role of transfusion in head and neck oncologic and reconstructive surgery. I will describe how this has evolved over time in my practice with self-reflection, PSQI, and finally to the ability to be open to change and listen to those who are junior to oneself. The process of initiating a change and following through on that change so it is integrated into one's practice is translatable into almost any process or protocols that we utilize. While I have chosen to discuss transfusion criteria, the same process is applicable to multiple other changes instituted over the years.

\section{Transfusion Criteria in the Early Days}

Head and neck ablative and reconstructive surgery is oftentimes associated with blood loss that warrants transfusion. The exact level at which patients require transfusion remains controversial. Early in my career, I established that a hematocrit level below $30 \%$ or a hemoglobin level below $10 \mathrm{~g} / \mathrm{dL}$ warranted transfusion. I based this decision on data from animal research dating back to the 1970s. In 1988, the National Institutes of Health consensus statement confirmed that "modern surgical and anesthetic practice" has been
Issue Theme Technologic Advancements in Head and Neck Reconstruction; Guest Editors: Sameep P. Kadakia, MD, and Sunishka M. Wimalawansa, MD, MBA
DOI https://doi.org/ 10.1055/s-0040-1721111. ISSN 0736-6825. 
guided by the belief that a hemoglobin value of less than $10 \mathrm{~g} / \mathrm{dL}$ or hematocrit value of less than $30 \%$ indicates the need for perioperative red blood cell transfusion. ${ }^{1}$ It was my belief that establishing a firm cutoff for transfusion was better than attempting to evaluate on an individual patient basis. While there was much literature in animal models that demonstrated that various cutaneous, fasciocutaneous, and myocutaneous flaps had improved survival when anemic, the translation of this information to the human model was lacking. ${ }^{2-4}$ Complications from red blood cell transfusions, while uncommon, can be devastating from both an infectious and an noninfectious process. Resource utilization is also a factor to take into account in our current health care model.

In 2007, a resourceful resident Dr. Zachary Solar undertook a literature review following an intensive care unit (ICU) rotation, which demonstrated that blood transfusions were associated with increased rates of infection, longer hospital stays, and increased mortality in critically ill patients. ${ }^{5-7}$ The increased threshold for transfusion in the ICU setting for these critically ill patients prompted an in-depth evaluation of our practice. All microvascular reconstructive surgeons are leery of a change in protocol or technique. The downside of a dead flap is a fear that oftentimes makes us dogmatic and unaccepting of change. To evaluate a change in our transfusion protocol, we analyzed in a retrospective fashion what the impact would be if we modified our transfusion threshold. We reviewed 54 free-flap patients transfused over the previous year and a half. Eighty percent of transfusions went to patients with hematocrit levels between 25 and 30\%. Multiple joint discussions with trauma surgeons, intensivists, and other faculty were undertaken. A consensus decision to decrease the transfusion criteria to a hematocrit level of $25 \%$ was made. The nonotolaryngology transfusion literature supported that the morbidity may in fact be less. Survival of free flaps in animal models supported the move and our retrospective analysis supported that it would be highly impactful on patient care and resource utilization. ${ }^{8-10}$

We then collected data over the next year and a half on patients with our new transfusion level. We compared these data with the data of immediate previous year and a half to evaluate the clinical significance, the number of units of blood not transfused, and the number of patients who did not receive a transfusion based on the new protocol. We were able to demonstrate that a restrictive transfusion protocol significantly lowered the transfusion rate without increasing complication rates. The only difference was a higher rate of fistulas and respiratory failure in the group that more liberally transfused. ${ }^{11}$ This transfusion protocol then became the paradigm for the next decade. During this time, transfusion protocols were not a topic of interest in the literature or at national meetings.

Almost 10 years later, Dr. James Azzi started his facial plastic and reconstructive microvascular fellowship in our department. During rounds, he remarked that at the institution where he completed his residency, the transfusion criteria were more restrictive, using a hematocrit level of $21 \mathrm{~g} / \mathrm{dL}$ to determine transfusion needs. This prompted much discussion and thought. Having already changed our trans- fusion criteria with no adverse effects and improvements in decreasing units of blood transfused, we reevaluated our protocol. We followed the same steps by examining the previous 2-year history of transfusions. We reviewed what the theoretical effect of a more restrictive transfusion level of $21 \%$ would have on the number of units and patients transfused. Forty-five percent of patients had a hematocrit less than $25 \%$ and received a transfusion. Of these, $80 \%$ of patients transfused never had a hematocrit less than $21 \%$. The potential impact was clinically significant for both the patients as well as the hospital system. Meetings were held between intensivists, trauma surgeons, and the department.

In March 2018, almost a decade after our first change, we again instituted a more restrictive transfusion protocol. The new hematocrit level was 21\%. Data were collected on 142 patients undergoing free tissue transfer and morbidity assessed. We were able to demonstrate that there was no increase in flap loss, fistula formation, pulmonary complications, or other patient morbidities. Only $23 \%$ of patients were transfused under our new restrictive guidelines. This was half the number that would have been transfused under our old criteria. Overall our transfusion rate for patients undergoing free tissue transfer evolved from approximately $75 \%$ of patients receiving a transfusion to $25 \%$ with a restrictive protocol. Our new restrictive guidelines have remained in place since then.

I have used this incorporation of increasingly restrictive transfusion criteria in a microvascular reconstructive practice to demonstrate how one can alter the paradigm of one's practice. Ideas for innovative approaches to patient care can arise on many levels. The ability to integrate the information from different sources and evaluate how it can influence one's practice is important. Analyzing what is currently being done and evaluating through multispecialty consultation which changes can be instituted, a plan to provide an innovative new management paradigm can be instigated. It is important to keep an open mind to ideas from those less experienced and perhaps most importantly from other specialties. An important first step is to analyze the data and evaluate if the paradigm shift in fact has been helpful or not. In our setting over the course of two decades, we have been able to alter our transfusion rate by greater than $60 \%$ in our patient population. The benefits to the patients in the system are significant. We have utilized this model to look at multiple other clinical and practice issues. These range from vasopressor use to ICU stays and many others.

\section{Conflict of Interest}

None.

\section{References}

$1 \mathrm{NIH}$ consensus statement available on perioperative red cell transfusion. Am J Public Health 1988;78(12):1588

2 Desyatnikova S, Winslow C, Cohen JI, Wax MK. Effect of anemia on the fasciocutaneous flap survival in a rat model. Laryngoscope 2001;111(4, Pt 1):572-575

3 Gatti JE, LaRossa D, Neff SR, Silverman DG. Altered skin flap survival and fluorescein kinetics with hemodilution. Surgery 1982;92(02):200-205 
4 Earle AS, Fratianne RB, Nunez FD. The relationship of hematocrit levels to skin flap survival in the dog. Plast Reconstr Surg 1974;54 (03):341-344

5 Hébert PC, Wells G, Blajchman MA, et al. A multicenter, randomized, controlled clinical trial of transfusion requirements in critical care. Transfusion requirements in critical care investigators, Canadian Critical Care Trials Group. N Engl J Med 1999;340 (06):409-417

6 Hill GE, Frawley WH, Griffith KE, Forestner JE, Minei JP. Allogeneic blood transfusion increases the risk of postoperative bacterial infection: a meta-analysis. J Trauma 2003;54(05):908-914

7 Hébert PC, Yetisir E, Martin C, et al; Transfusion Requirements in Critical Care Investigators for the Canadian Critical Care Trials Group. Is a low transfusion threshold safe in critically ill patients with cardiovascular diseases? Crit Care Med 2001;29(02):227-234
8 Taniguchi Y, Okura M. Prognostic significance of perioperative blood transfusion in oral cavity squamous cell carcinoma. Head Neck 2003;25(11):931-936

9 Szakmany T, Dodd M, Dempsey GA, et al. The influence of allogenic blood transfusion in patients having free-flap primary surgery for oral and oropharyngeal squamous cell carcinoma. $\mathrm{Br} \mathrm{J}$ Cancer 2006;94(05):647-653

10 Fenner M, Vairaktaris E, Nkenke E, Weisbach V, Neukam FW, Radespiel-Tröger M. Prognostic impact of blood transfusion in patients undergoing primary surgery and free-flap reconstruction for oral squamous cell carcinoma. Cancer 2009;115(07): 1481-1488

11 Rossmiller SR, Cannady SB, Ghanem TA, Wax MK. Transfusion criteria in free flap surgery. Head Neck Surg 2010;142(03): 359-364 